\title{
Caracterización de la agroindustria artesanal y atributos de la valoración \\ en consumidores de queso bola de Ocosingo, Chiapas ${ }^{\star}$
}

\section{Characterization of artisanal agro-industry and valuation aspects in consumers of queso bola of Ocosingo, Chiapas}

Recibido: 12 de Noviembre de 2018

Revisado: 2 de diciembre de 2018

Aprobado: 10 de diciembre de 2018

* Artículo producto del proyecto de investigación caracterización de la agroindustria artesanal en Chiapas, México. Cómo citar este artículo: Espejel, A. (2019). Caracterización de la agroindustria artesanal y atributos de la valoración en consumidores de queso bola de Ocosingo, Chiapas. Revista CIFE: Lecturas de Economía Social, 20(33), 137-155. DOI: https://doi.org/10.15331/22484914.4881

** Profesor investigador del posgrado en Ciencia y Tecnología Agroalimentaria, Departamento de Ingeniería Agroindustrial, Universidad Autónoma Chapingo. Correo electrónico: anastacio.espejel@gmail.com 


\section{Resumen}

El queso bola de Ocosingo, Chiapas es un queso emblemático y tradicional que posee características típicas y diferenciadas. La normativa restringe los quesos elaborados con leche cruda. Existe una baja valoración del consumidor con respecto a los quesos artesanales. Los objetivos de esta investigación fueron medir la adopción de innovaciones y su relación con las ganancias en queserías y aspectos de valoración del consumidor. Se seleccionaron seis queserías y se midió el nivel de adopción de innovaciones y utilidad. Se aplicaron cien encuestas a consumidores de Ocosingo, Chiapas y Texcoco, México. Las innovaciones de mayor adopción fueron la administración y estandarización del proceso; la utilidad y estrategia comercial son variables que impactan en mejores ganancias. Los consumidores de mayor ingreso y escolaridad tienen mayor disposición a pagar (DAP) por un queso artesanal, los atributos más valorados son etiqueta, empaque y sensoriales. Una mayor difusión del producto en consumidores con mayor DAP resultaría en mejores ganancias.

Palabras clave: innovación, agroindustria artesanal, valoración.

Clasificación JEL: L2, M1 1

\section{Abstract}

The queso bola of Ocosingo, Chiapas is a traditional cheese that has typical and distinctive characteristics. The regulation restricts cheeses made with raw milk. There is low consumer valuation with respect to artisan cheeses. The objective of this research was to measure the adoption of innovations and its relationship with the profits in cheese factories and aspects of consumer valuation. Six cheese factories were selected and the level of adoption of innovations and profits was measured. One hundred surveys were applied to consumers in Ocosingo, Chiapas and Texcoco, Mexico. The innovations of greater adoption were administration and standardization of the process; usefulness and commercial strategy are variables that impact on better profits. Consumers with higher income and schooling have more willingness to pay (WTP) for an artisanal cheese, the most valued traits are label, packaging and sensory. A greater diffusion of the product in consumers with higher WTP would result in better profits.

Keywords: Innovation, artisanal agro-industry, valuation.

Classification JEL: L2, M1 1 


\section{Introduction}

Los alimentos tradicionales (TFP) son considerados elementos importantes de la cultura, identidad y patrimonio gastronómico. Contribuyen al desarrollo, diversificación y sostenibilidad de muchas áreas rurales, deteniendo el éxodo. La Unión Europea es la región líder en la investigación y comercialización de alimentos tradicionales. Europa es la región donde la cultura y el reconocimiento social de estos productos han sido consecuencia de diversos trabajos de investigación y difusión de las bondades y atributos de este tipo de productos. La caracterización del alimento, la protección jurídica para evitar el plagio y la implementación de modelos que certifiquen la autenticidad del producto son esfuerzos que dieron como resultado la comercialización legal de productos tradicionales.

La producción de TFP en Europa se lleva a cabo principalmente por las pequeñas y medianas empresas (pyme), y estos productos se comercializan mayormente mediante una diferenciación o protección, entre las principales tenemos a las denominaciones de origen, indicaciones geográficas y marcas colectivas (Guerrero, Claret, Verbeke, Sulmont y Hersleth, 2016; Kristbergsson y Oliveira, 2016; Trichopoulou, Vasilopoulou, Georga, Soukara y Dilis, 2006).

En México, los quesos genuinos elaborados a partir de leche cruda son los productos más emblemáticos y representativos de un alimento tradicional. Las empresas agroindustriales en donde se produce este tipo de queso están por lo regular aglomeradas en espacios rurales donde comparten recursos naturales, geográficos y agroecológicos que confieren atributos específicos a la materia prima (leche), por consiguiente, al queso, y representan un potencial para su diferenciación con respecto a los quesos de imitación e industrializados.

Los eslabonamientos dentro de este tipo de cadenas son cortos y con un nivel de proximidad elevado, la comercialización se realiza mediante modelos de circuitos cortos, que permite a estos quesos insertarse de manera eficiente en los mercados locales y en muy pocas ocasiones nacionales, disminuyendo los costos de transacción, incluso se habla de que son competitivos aun con todas las asimetrías en el acceso y valoración del consumidor. La empresa quesera tradicional está compuesta por micro y pequeñas empresas, con una organización productiva, dinámica y lógica de producción propia; se caracteriza por la incorporación de mano de obra familiar, integración de recursos locales y aplicación de tecnología e innovaciones acordes a las necesidades de producción y demanda del mercado donde se insertan (Cervantes, Villegas, Gesín y Espinoza, 2008; Solís, 2018).

El consumo de quesos en México es elevado en todos los niveles socioeconómicos, promovido en parte por la diversidad de quesos mexicanos tradicionales (más de 40), entre 
ellos panela, quesillo, asadero, chihuahua, cotija, adobera, crema de Chiapas, bola, etc. El queso artesanal mexicano es un alimento con historia y tradición, forma parte del legado cultural del país; son quesos tan diversos como heterogéneos en calidad, que compiten desventajosamente con quesos industriales y de imitación o con ingredientes sustitutos y análogos (Hervás, 2012).

Los consumidores, actualmente, demandan sabores únicos y comidas típicas, sin culpa de cocinar y comer, y una dieta cada vez más focalizada en el cuidado de la salud y acorde a sus necesidades y preferencias individuales (Sarkar y Costa, 2008). Los consumidores de este tipo de productos aceptan algunas innovaciones (etiquetado y envase) que no alteran la calidad sensorial y mejoran la apariencia y vida útil del producto; otro grupo de innovaciones están orientadas a productos más sanos y seguros, por ello, el sector agroindustrial invierte a través de ingredientes seleccionados, materia prima de mejor calidad y trazabilidad. Sin embargo, se busca que en este tipo de productos, las innovaciones no comprometan las propiedades sensoriales de los productos alimenticios tradicionales, como en los quesos mexicanos genuinos tradicionales (Kühne, Vanhonacker, Gellynck y Verbeke, 2010).

Los productores de alimentos tradicionales enfrentan el desafío de mejorar la seguridad y conveniencia de sus productos, de acuerdo con la demanda de los consumidores mediante diferentes tipos de innovaciones que permiten mantener o expandir su influencia, en un entorno altamente competitivo y globalizado. Un mejor entendimiento de los consumidores, de sus creencias, percepciones y expectativas es esencial para el desarrollo de productos e introducción de innovaciones, así como la implementación de acciones de marketing exitosas (Kristbergsson y Oliveira, 2016).

Dentro de los quesos genuinos mexicanos, quizá uno de los más emblemáticos es el queso bola de Ocosingo, Chiapas, el cual posee características tecnológicas únicas, externamente posee una cubierta que sirve como empaque natural a base leche descremada, y el centro es un queso crema madurado al menos por 21 días, de acuerdo con las reglas de uso de la marca colectiva. Un problema es la normativa que no permite la comercialización de quesos elaborados con leche cruda (NOM-243-SSA1-2010) y la baja valorización del consumidor con respecto a los atributos de estos productos. Los objetivos de esta investigación fueron analizar las empresas queseras tradicionales, a fin de evaluar las innovaciones adoptadas y su relación con la utilidad y el análisis de los consumidores y factores que influyen en la decisión de compra de este producto, para proponer estrategias que incrementen el posicionamiento de los quesos artesanales. 


\section{Metodología}

\subsection{Caracterización de la agroindustria (AI)}

Se diseñaron y aplicaron encuestas estructuradas a las queserías: El Dorado, Queshil, Laltic, Productos Ocosingo, Lácteos Maya y Santa Rosa, con la finalidad de caracterizar y analizar la adopción de tecnología e innovaciones con las que cuentan cada una. La encuesta tuvo cuatro categorías principales: 1) aspectos generales del productor, 2) organización, 3) dinámica de la agroindustria y 4) dinámica de innovaciones.

\subsection{Adopción de innovaciones}

Los datos obtenidos de las encuestas fueron sistematizados en Excel; se calculó el índice de adopción de innovaciones (INAI), en el cual se expresan las innovaciones que realiza cada productor dentro del total de innovaciones, utilizando la siguiente ecuación (1) (Muñoz, Altamirano, Aguilar, Rendón y Espejel, 2007).

$$
I N A I=\sum_{i=1}^{j}\left[\frac{\text { innovaciones realizadas por el innovador } i}{\text { máximo número de innovaciones por los productores } j}\right] * 100
$$

\subsection{Velocidad de adopción de innovación}

Con el propósito de conocer la trayectoria de las innovaciones implementadas por las agroindustrias queseras, se calculó a través de los resultados de las encuestas, la velocidad de adopción de innovación con la siguiente ecuación (2) (Rendón, Aguilar, Muñoz y Altamirano, 2007).

$$
V A=\frac{a \text { ño de registro de la primera innovación }- \text { año más reciente de adopción }}{\text { porcentaje de produtores adoptantes por año }}
$$

\subsection{Análisis económico}

Se realizó considerando los costos generados con la producción y los ingresos totales en la unidad productiva (cantidad de queso producido multiplicada por el precio corriente). Los beneficios fueron estimados con la ecuación (3) (Espejel, Barrera, Herrera y Cuevas, 2016).

$$
B=\sum_{t=1}^{n} I T-\sum_{i=1}^{n} C T
$$


Donde:

$\mathrm{B}=$ beneficios o ganancias económicas de la actividad.

IT = sumatoria de los ingresos totales de la unidad productiva de 1 hasta $\mathrm{n}$.

$\mathrm{C}=$ Sumatoria de los costos de producción incurridos en la unidad productiva de 1 hasta $n$.

\subsection{Análisis estadístico}

La información obtenida de las encuestas fue organizada y registrada en una base de datos de Excel, para su posterior análisis. Primero se realizó un análisis de correlación de Pearson para identificar la asociación entre variables (calidad, estandarización del proceso, manejo de residuos, estrategia comercial y utilidades) y su relación con las ganancias económicas.

Se realizó un análisis de componentes principales (ACP) para identificar las variables que influyen en el posicionamiento de las empresas queseras. Los datos se analizaron con el paquete estadístico XLSTAT 2014.5.03.

\subsection{Caracterización del consumidor de queso bola}

Se aplicaron cien encuestas a consumidores del queso bola en dos lugares: Feria del queso bola 2017 en Ocosingo, Chiapas y Feria de la Cultura Rural en Texcoco, estado de México. Los criterios de selección fueron ser consumidor del queso bola y mayoría de edad. También se incluyeron variables que permitieron definir el perfil sociodemográfico de los consumidores potenciales del queso (edad, nivel de ingresos, ocupación, lugar de origen, sexo, estado civil, etc.) y hábitos de consumo como: percepción del queso, frecuencia de consumo, marca preferida, lugar de compra, precio del producto.

Se realizó un análisis de regresión logística para obtener las variables de mayor influencia en relación con la disposición a pagar por el queso bola, y un análisis de componentes principales (ACP) para identificar las variables que más influyen en la decisión de compra e identificar los atributos que el consumidor valora. Los datos se analizaron con el paquete estadístico XLSTAT 2014.5.03. 


\section{Resultados y discusión}

\subsection{Problemática de las empresas queseras}

Uno de los principales problemas identificados por el $60 \%$ de los productores del queso bola es el desabasto de leche, principalmente en la época de estiaje, este hecho afecta a aquellos productores que no producen su materia prima y dependen exclusivamente de la oferta y demanda, mientras otros se ven en la necesidad de comprar leche en polvo $(15 \%)$, afectando así a la genuinidad y tipicidad del queso bola.

Pese a la importancia cultural del queso bola, existe de manera constante la presión sobre los queseros por modificar la tipicidad de sus productos, impulsado por las instituciones gubernamentales que establecen requerimientos sobre la calidad en los procesos de producción de quesos: la NOM-243-SSA1- 2010; la NOM-251-SSA-2009, la Secretaría de Salud, la NOM-051-SCFI-SSA1-2010 y la NMX-713-COFOCALEC-2005, oficialmente son las normativas que regulan la calidad mínima necesaria para elaborar y comercializar los quesos producidos en México. Pese a que ya existen estudios en quesos tradicionales genuinos mexicanos, donde se demuestra la inocuidad del queso y la disminución del microbiota de estos, como es el caso del queso cotija e incluyendo al queso bola, siendo quesos elaborados con leche cruda; falta entendimiento y sensibilización de las instituciones correspondientes para dar la importancia necesaria a estos quesos (Escobar, Hernández y Gaspar, 2012; López, 2013; Hernández, 2014).

Los cambios en los hábitos de consumo y el ritmo de vida de la sociedad resultan en la alteración de la producción de alimentos a nivel mundial, es decir, cambian por completo las fronteras alimentarias, y se homogeniza y monopoliza la alimentación de manera industrial y también la población demanda productos con propiedades funcionales específicas (Solís, 2018; Vendruscolo, Tomé da Cruz y Schneider, 2016).

Los consumidores son el factor más importante, cada vez son más los que prefieren productos genuinos tradicionales y prestan mayor atención a la procedencia geográfica de los productos, y se preocupan por determinadas características presentes en los productos que adquieren. En ocasiones, el lugar de origen sugiere a los consumidores que el producto tendrá una calidad o características sensoriales específicas que confieren atributos de mayor valoración para un grupo de consumidores.

A menudo, los consumidores están dispuestos a pagar más por estos productos, favoreciendo mercados específicos para productos con determinadas características vinculadas a su lugar de origen (Rizo et al., 2013). 


\subsection{Adopción de innovaciones en la AI quesera}

La innovación en productos artesanales debe introducirse de manera cuidadosa y creativa, ya que un cambio en el proceso de estos productos podría no ser el más adecuado para el consumidor que valora lo artesanal y típico de estos productos tradicionales. Para el análisis del índice de adopción de innovaciones se consideraron las categorías: producción (calidad), estandarización del proceso, manejo de residuos, estrategia comercial y utilidades. En la figura 1 se observa que la categoría de mayor adopción de innovación durante los últimos años para AI son: administración, ya que el $100 \%$ de los productores cuentan registros manuales de sus operaciones y estandarización del proceso, esto porque el $50 \%$ de los productores respetan lo estipulado en las reglas de uso para la marca colectiva "queso bola de Ocosingo, Chiapas". Para esta última categoría se consideraron cuatro puntos: leche cruda, tiempo de maduración del queso, sin aditivos (excepto sal y cuajo) y la elaboración del forro.

Las categorías que se encontraron por debajo del $50 \%$ de adopción corresponden a calidad, estrategia comercial y manejo de residuos, por lo que se atribuye a un bajo interés de los productores en estas innovaciones. En el caso de la estrategia comercial es necesario profesionalizar y promover de manera más eficiente el producto, de manera paralela es necesario informar al consumidor sobre las bondades del queso bola más allá del consumo y del aspecto tangible. La diferenciación a partir de etiquetas con información sobre aspectos culturales del producto podría contribuir a una mayor diferenciación.

Figura 1. Medias de las diferentes categorías para adopción de innovaciones en la agroindustria

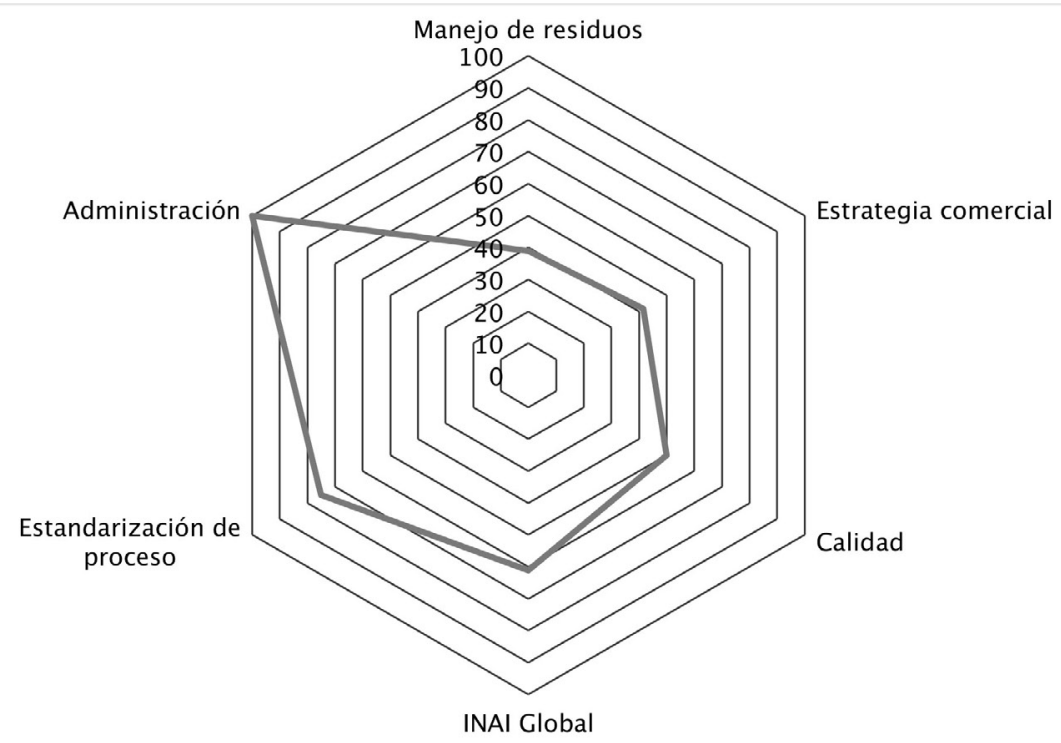

Fuente. Elaboración propia (2017) 
Con respecto a los atributos sensoriales, el queso bola posee características únicas, la forma en que lo elaboran, el proceso de maduración y los recursos naturales asociados, así como la ubicación geográfica, confieren rasgos típicos: olor, sabor, color, consistencia que lo diferencian de los demás quesos madurados. Sin embargo, la valoración que el consumidor le da al producto es baja aunado a débiles estrategias de promoción de los productores.

El queso es elaborado por un grupo reducido de queseros artesanales, quienes han heredado y conservado el saber hacer de este queso por generaciones, conservando la identidad, tipicidad y tradicionalidad. Por otro lado, en los mercados locales se comercializa queso bola de diversas calidades, con variaciones que afectan la tipicidad, tales como la disminución del tiempo de maduración, forro más fresco y en casos extremos la adición de leche en polvo, resultando un producto más fresco, y sensorialmente diferente y perdiendo parte de lo genuino y típico. Esto coincide con el porcentaje bajo en la adopción de innovaciones del queso, pues la mayoría de las queserías no respetan las reglas de uso de la marca colectiva para la elaboración del queso bola, lo que demerita su valoración (Cervantes et al., 2008; Pomeón, 2011).

\subsection{Velocidad de adopción de innovación de la AI}

Se calculó para conocer velocidad con la que los productores aprenden y adoptan innovaciones durante el tiempo, lo cual está relacionado directamente con la capacidad de adaptación de las empresas al entorno y tendencias de consumo. En la figura 2 se destacan dos etapas, la primera que va de 1969 a 2012 y la segunda que va de 2012 a 2017.

Figura 2. Velocidad de innovación de la AI del queso bola de Ocosingo, Chiapas

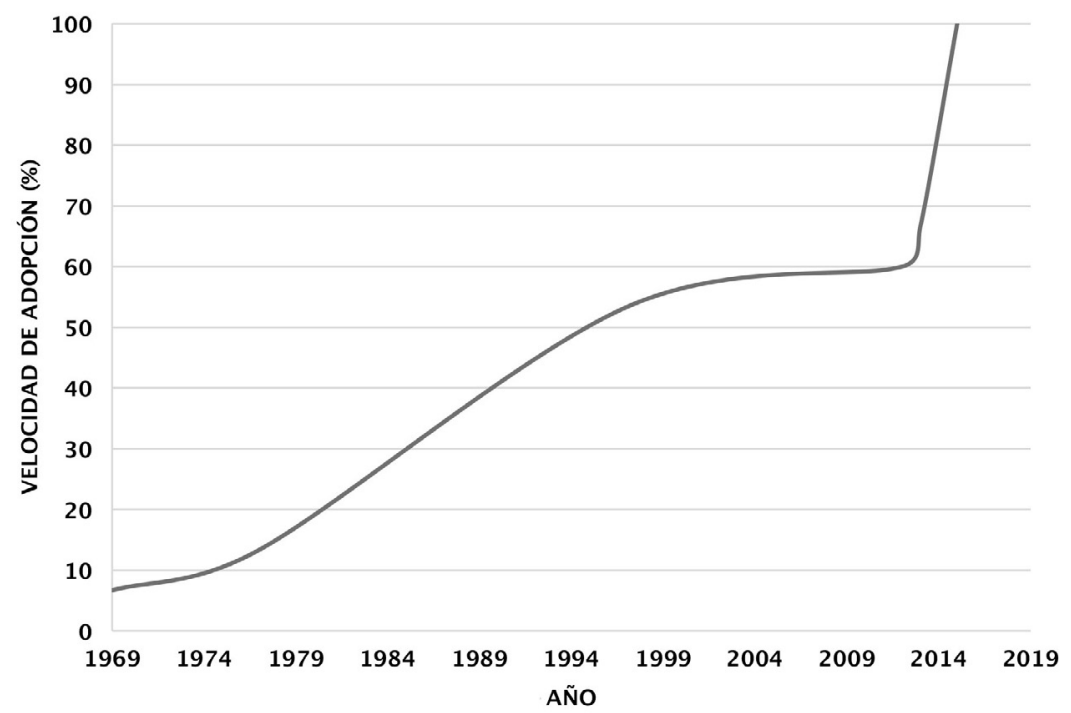

Fuente. Elaboración propia (2017) 
En la primera fase, se aprecia que las innovaciones que se han introducido se relacionan con la mejora del proceso y la integración de equipos y trazabilidad de la materia prima, es decir, se puede asociar como una etapa de fortalecimiento de lo típico y tradicional. En la segunda etapa, se aprecia una mayor rapidez en adoptar innovaciones relacionadas con mayores exigencias del mercado y entorno, tales como las buenas prácticas de producción, introducción de innovaciones de cuidado ambiental, como el manejo del lactosuero y la necesidad de vincular el queso con mercados de mayor valor, implicando mejorar la estrategia comercial, utilizando las redes sociales y mayor participación en ferias para promocionar el queso bola.

\subsection{Análisis económico de las queserías del queso bola}

El análisis económico en las agroindustrias (figura 3) es un indicador que muestra de manera general las utilidades derivadas de la producción quesera, y puede representar la continuidad o no de la producción de este queso. El análisis indica que el $33 \%$ de las queserías tienen mayor ingreso con respecto a los costos asociados a la producción del queso, por lo que podría asumirse una mayor rentabilidad, el resto puede ser que presente utilidades negativas, sin embargo, es posible que para este grupo de queseros la actividad represente una parte complementaria.

Figura 3. Análisis económico de las queserías del queso bola de Ocosingo, Chiapas

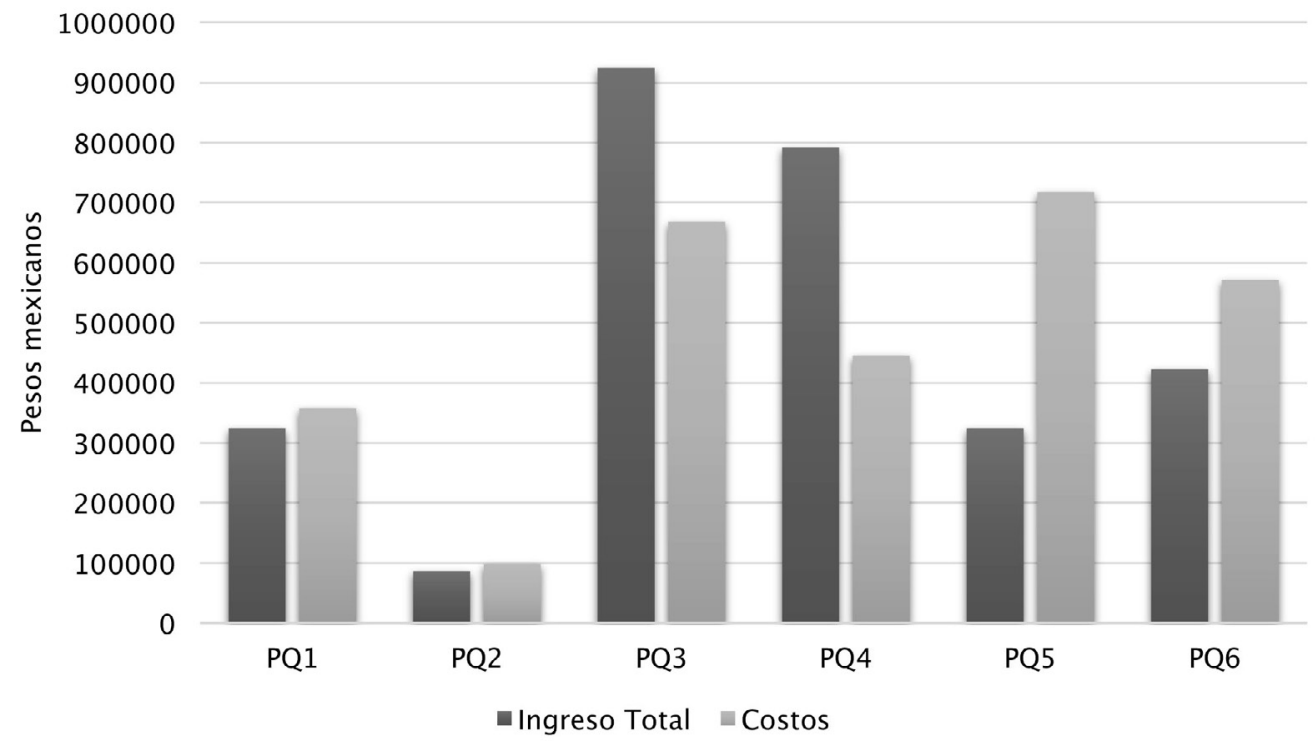

Fuente. Elaboración propia (2017)

PQ1 (El Dorado), PQ2 (Queshil), PQ3 (Laltic), PQ4 (Productos Ocosingo), PQ5 (Lácteos Maya) 
Las queserías con mayores utilidades están vinculadas a mercados más estables y de mayor valor, por lo que el precio al que comercializan su producto es más alto, en comparación con el resto de las queserías quienes muestran incluso pérdidas. La quesería (PQ3) posee un nivel de integración elevado y, por lo tanto, poseen mayor control sobre la materia prima, esto confiere una mayor calidad del queso. Por otro lado, la PQ4, no produce su propia leche, además cuenta con un mercado específico, el cual ya es garantía para la venta de sus quesos (por ejemplo: Aeropuerto Internacional Ángel Albino Corzo, Chiapa de Corzo, Chiapas).

Derivado del análisis de componentes principales (ACP), se obtuvo que con dos componentes se explica el $78 \%$ de la variabilidad de los datos $(\mathrm{KMO}=0.5)$, en la figura 4 se aprecia que las variables analizadas se distribuyen en dos grupos con alta afinidad al interior de ellos, en el primer grupo las variables de mayor peso son la estandarización del proceso, calidad y manejo de residuos (proceso), en el segundo grupo se ubica la estrategia comercial y utilidades, lo cual sugiere que aquellas empresas que ponen mayor énfasis en el mercado y los consumidores tienen mejores utilidades.

Figura 4. Análisis de componentes principales vinculados a las variables de adopción e innovación en seis queserías en Ocosingo, Chiapas

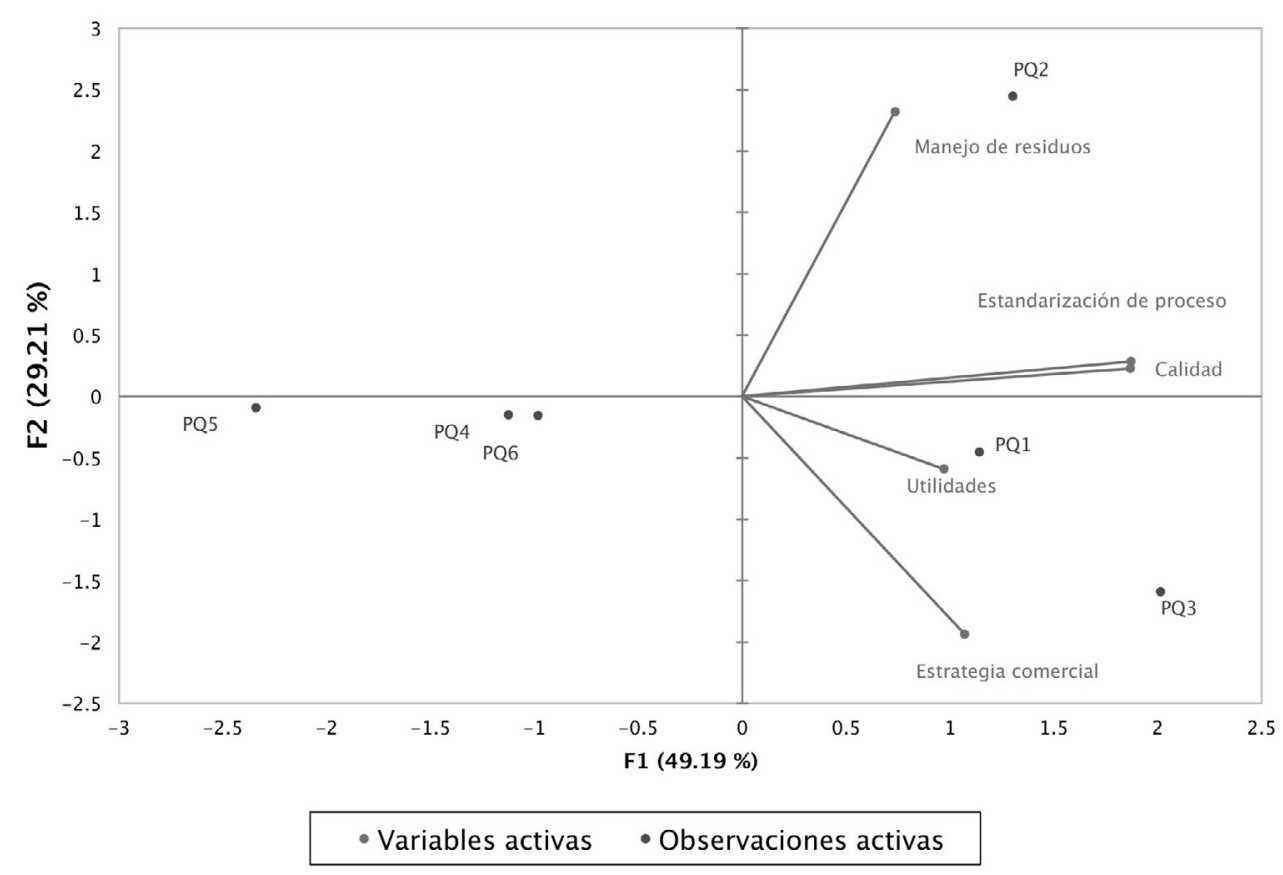

Fuente. Elaboración propia

PQ1 (El Dorado), PQ2 (Queshil), PQ3 (Laltic), PQ4 (Productos Ocosingo), PQ5 (Lácteos Maya) y PQ6 (Santa Rosa) 
La posición competitiva de las empresas queseras con respecto a los dos componentes principales permite diferenciar que en el componente 1 (eje $\mathrm{X}$ ) las variables de mayor peso son calidad y estandarización del producto; y con respecto al componente 2 (eje Y) la variable de mayor peso es la del manejo de residuos (tabla 1), siendo los factores que dan mayores ganancias en las queserías.

Tabla 1. Cargas factoriales de los componentes

\begin{tabular}{|lcc|}
\multicolumn{1}{|c}{ Variable } & $\mathbf{F 1}$ & F2 \\
\hline Calidad & $\mathbf{0 . 9 4 7}$ & 0.110 \\
\hline Estandarización de proceso & $\mathbf{0 . 9 4 4}$ & 0.087 \\
\hline Manejo de residuos & 0.371 & $\mathbf{0 . 9 0 4}$ \\
\hline Estrategia comercial & 0.541 & -0.755 \\
\hline Utilidades & 0.491 & -0.231 \\
\hline
\end{tabular}

Fuente. Elaboración propia con datos de campo (2017)

De acuerdo con los resultados, en este grupo de queserías las ganancias (utilidades) tiene relación directa con la adopción de innovaciones, lo cual sugiere que las innovaciones evaluadas contribuyen de manera sustancial con este modelo de queserías, principalmente las relacionadas con la estrategia comercial, manejo de residuos y conservación del proceso de producción; incluso las de mejor posicionamiento muestran fuerte relación con una estrategia comercial basada en la identificación de necesidades del consumidor y nuevos mercados.

Las variables relacionadas con la estandarización del proceso y calidad están altamente correlacionadas e influyen en la posición de las queserías. Un aspecto relevante es que todas las queserías no consideran importante el análisis del consumidor y del mercado, aunque ello pueda implicar una mejor posición en el mercado.

Con relación a las reglas de uso de la marca colectiva, buena parte de los queseros no las respeta, obteniendo calidades distintas en los productos, demostrando así la baja organización de los productores del queso bola, y por lo tanto una marginal alternativa de que este producto pueda acceder a otro tipo de protección o indicación geográfica, pese a ser un buen candidato. 


\section{Caracterización del consumidor actual y potencial del queso bola}

\subsection{Características sociodemográficas de los consumidores}

Es posible que las queserías de tamaño pequeño y mediano se vean limitadas en la fortaleza de la relación que establecen con sus clientes, y sobre todo que entiendan sus necesidades. Los resultados derivados del análisis indican que los consumidores son personas adultas, en edad laboral, en un rango de edad entre los 20 a 79 años, de los cuales el $40 \%$ poseen un nivel de escolaridad básica y media superior, un $50 \%$ con estudios universitario y un $10 \%$ con estudios de posgrado.

\subsection{Características socioeconómicas de los consumidores}

Con respecto al análisis socioeconómico de los consumidores, se clasificaron en tres estratos, en relación con el nivel de ingresos mensuales que expresaron tener, lo cual se asocia con la ocupación de estos. Los resultados se muestran en la tabla 2, y sugieren que el $50 \%$ de los encuestados tienen un grado universitario; el $30 \%$ de la población se encuentra en el estrato 1, esto se asocia a que muchos perciben un salario muy bajo o no ejercen su profesión, lo cual limita la capacidad de compra. En el estrato 3, con un $7 \%$ de participación se encuentran los consumidores con estudios de posgrado, ya que cuentan con trabajos estables o son empleados del Gobierno, quienes perciben un salario mayor a los consumidores de los otros estratos.

Tabla 2. Clasificación por estratos de acuerdo con el nivel de ingresos mensual y escolaridad de los consumidores

\begin{tabular}{|c|c|c|c|}
\hline Variable & $\begin{array}{l}\text { Estrato (bajo) } \\
(<\$ 5,000 / \text { mes })\end{array}$ & $\begin{array}{c}\text { Estrato } 2 \text { (medio) } \\
(\$ 5,001-\$ 10,000 / \text { mes })\end{array}$ & $\begin{array}{l}\text { Estrato } 3 \text { (alto) } \\
(>\$ 10,000 / \mathrm{mes})\end{array}$ \\
\hline Con posgrado (\%) & & 3 & 7 \\
\hline Estudios superiores $(\%)$ & 30 & 13 & 7 \\
\hline Media superior $(\%)$ & 19 & 6 & \\
\hline Básica (\%) & 10 & 1 & 3 \\
\hline Edad (años) & 34 & 38 & 43 \\
\hline \multicolumn{4}{|l|}{ Consume queso por: } \\
\hline Apariencia (\%) & 46 & 22 & 12 \\
\hline Inocuidad (\%) & 80 & & 20 \\
\hline Identidad $(\%)$ & 54 & 29 & 18 \\
\hline Tradición (\%) & 65 & 22 & 13 \\
\hline
\end{tabular}

Fuente. Elaboración propia con base a datos de campo (2017) 
En relación con la edad, los consumidores del estrato alto se caracterizan por tener mayor edad comparado con los otros dos estratos, lo cual repercute en decisiones más sólidas al momento de elegir los productos a consumir, principalmente los del tipo tradicional. Con respecto a la inocuidad del producto, los consumidores del estrato bajo y alto lo consideran importante. El consumo de este producto asociado a la identidad, tradición y apariencia es importante para los tres estratos, lo cual significa que el consumidor de este queso está informado sobre aspectos intangibles asociados al producto y lo consume por sus características de tipicidad.

\subsection{Variables que inciden en la disposición a pagar}

Con base al análisis de regresión logística, en la figura 5 se observan las variables de mayor influencia en relación con la disposición a pagar por el queso bola. Las variables de mayor incidencia son la edad, escolaridad y nivel de ingresos del estrato 3 (mayor ingreso y mayor nivel de estudios). Estas variables son las que influyen en una mayor disposición a pagar por un queso artesanal, lo cual está relacionado directamente con un mayor nivel de ingreso y nivel educativo, esto se podría asociar con un mayor conocimiento y valorización de un producto tradicional, considerando las características asociadas, tales como recursos naturales, cultura, tradición y saber hacer.

Figura 5. Variables que influyen para la disposición a pagar por el queso bola

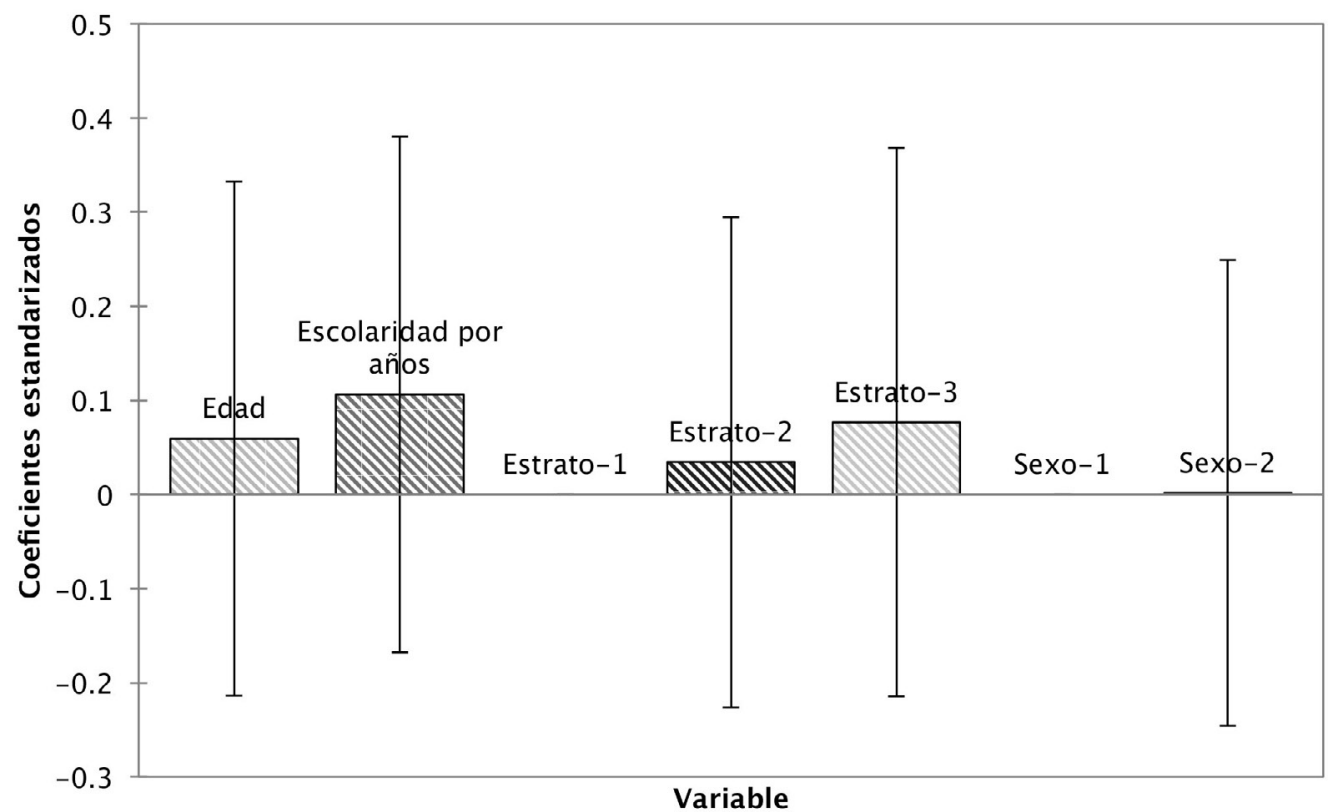

Fuente. Elaboración propia con base a datos de campo (2017) 
Existe una clara ventaja competitiva de los alimentos tradicionales con respecto a los industriales, ya éstos son alimentos ofrecen comodidad a un precio económico para la mayoría de los consumidores; son productos que están estandarizados, ofrecen calidad constante todos los días y la mayoría son quesos frescos. Generalmente se producen a gran escala por grandes empresas en cualquier lugar del mundo y los productores pueden tener casi el mismo producto final.

Caso contrario con los quesos tradicionales, como el queso bola de Ocosingo, Chiapas, que son productos hechos a mano y producidos en un territorio de origen mediante el uso de recursos naturales específicos, condiciones climáticas, raza del animal adaptada al entorno y, por tanto, son testimonio de la historia, cultura y estilo de vida de la comunidad rural y entorno geográfico en donde se producen. Este tipo de queso se caracteriza por sus sabores intensos y distintivos, con una variabilidad razonablemente alta, incluso dentro de la variedad del mismo queso (Licitra, 2010).

Un grupo reducido de consumidores que comparte características específicas valora este tipo de productos, sin embargo, existe evidencia acerca del desconocimiento para la mayoría de los consumidores sobre los atributos de los quesos tradicionales diferenciados por su calidad de origen. Es decir, los consumidores que adquieren alimentos similares a los quesos mexicanos tradicionales siguen sin conocer su diversidad o existencia, como es el caso del queso bola de Ocosingo (Agudelo et al., 2016).

Para asociar los atributos que el consumidor valora del queso bola y los grupos de consumidores, se realizó un ACP, donde se obtuvo que dos componentes explican el $75 \%$ de la variabilidad de los datos $(\mathrm{KMO}=0.6)$ los factores que consideran los consumidores en el consumo de este queso son las variables como el sabor, aroma y textura (características sensoriales), en el segundo la etiqueta y empaque, en el tercero el nivel de ingresos, escolaridad y lugar de residencia; por otro lado, el precio, género y edad no son atributos relevantes (figura 6).

La posición de los consumidores con respecto a los dos componentes principales del ACP permite diferenciar en el componente 1 (eje $\mathrm{X}$ ) las variables de mayor peso que son aroma, textura y sabor; con respecto al componente 2 (eje Y) empaque y etiquetado.

En los últimos años se ha observado un creciente interés de los consumidores en productos alimenticios más diferenciados, en este contexto, los TFP representan una alternativa de consumo saludable y esto se asocia al hecho de que los consumidores son cada vez más reacios a los riesgos inducidos por la innovación alimentaria como resultado de la intervención humana; sin embargo, el desarrollo de TFP es necesario para la supervivencia del producto en un mercado global competitivo (Stolzenbach, Bredie y Byrne, 2013; Vanhonacker et al., 2013) 
Figura 6. Componentes principales vinculados a las variables que influyen en la decisión de compra en los consumidores

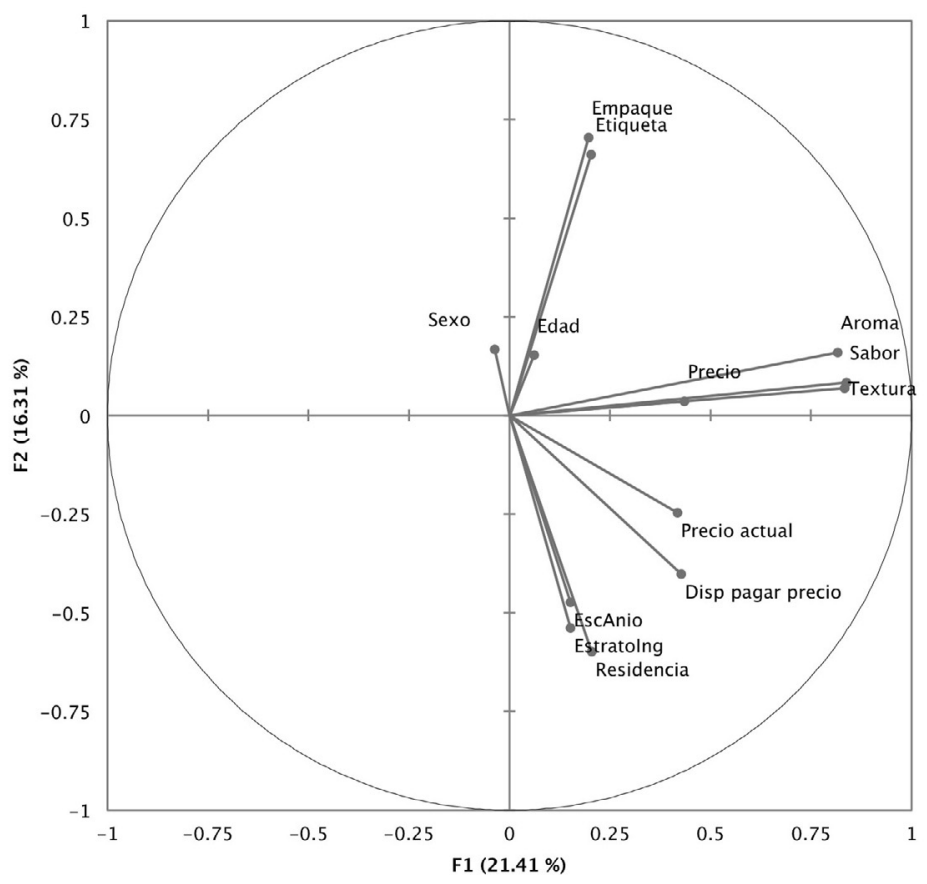

Fuente. Elaboración propia (2017)

Los TFP representan un segmento en crecimiento en el mercado alimentario, sin embargo, para mantenerse en el mercado actual, deben innovar y mejorar la forma de llegar a los consumidores, pero más importante es informar al mercado sobre los atributos de este tipo de productos. Cuanto más estrictamente los consumidores definen los alimentos tradicionales, más influye su aceptación en el impacto percibido de la innovación en el carácter tradicional del producto alimenticio (Vanhonacker et al., 2013).

La aceptación por parte de los consumidores de las etiquetas con garantía de origen y mayor variedad de productos corresponde a los esfuerzos del sector para ingresar a nuevos mercados y mejorar la comercialización de sus productos alimenticios tradicionales (Kühne et al., 2010).

Un factor importante para la aceptación de innovaciones y que beneficien a los productores del queso bola implica reforzar las características tradicionales del producto (por ejemplo, una etiqueta que garantice el origen) o que brinden beneficios al mejorar los atributos negativos asociados con características tradicional de los alimentos (por ejemplo, resaltar que es un producto madurado, genuino, sin adición de aditivos). 
Por otro lado, el productor tradicional debe tener una comprensión clara de las características que los consumidores buscan en el TFP (Kühne et al., 2010). Ya que el consumidor exige una relación más cercana con el productor de su comida, ya sea real (por ejemplo, la compra directa en el lugar de origen), o imaginaria (a través de un etiquetado con datos de su origen, elaboración e identidad), pues simbólicamente estos datos le representen cualidades intangibles y ayudan a compensar la distancia entre el consumidor del producto y la fuente del alimento (Bessiere, 1998).

Tal es el caso del estrato 3, que tienen la disposición de pagar mejor el queso bola, además otra característica singular es que son consumidores que difícilmente pueden acceder al producto en su lugar de origen, por lo que sería necesario trabajar con los productores en mejorar la información plasmada en la etiqueta, y que ellos mismos valoricen más su producto, ya que la mayoría o vende sin etiquetas, demeritando así la calidad de este producto tradicional.

\section{Conclusiones}

Existen posibilidades de introducir innovaciones en el queso bola de Ocosingo, Chiapas, sin disminuir su principal ventaja competitiva como sus rasgos característicos de la forma de elaboración, apariencia, y en especial las características relacionadas con la autenticidad y genuinidad del producto. Tal sería el caso de mejorar la etiqueta principalmente y de preservar las características de elaboración del queso, respetando lo que marcan las reglas de uso de la marca colectiva, para mantener la calidad de los quesos (maduración, forrado oreado, sin otros aditivos distintos al cuajo y sal).

Un estrato de los consumidores con mayor nivel de estudios y mayor nivel de ingresos son los que se identifican en mayor medida con los productos tradicionales, además de mostrar una mayor disposición a pagar por este tipo de queso.

Las variables que los consumidores consideran para adquirir el queso tienen que ver con tres grupos, en el primero se ubica el empaque y la etiqueta, en el segundo el aroma, textura y sabor y en el último grupo se encuentran variables como el precio, la escolaridad, estrato de ingreso y la residencia. Una estrategia que se puede implementar, derivada de este trabajo, fue identificar posteriormente nichos de mercado con este tipo de características. 


\section{Referencias}

Agudelo, M. A., Cesín, A. y Thomé, H. (2016). Alimentos emblématicos y turismo. La vinculación del queso bola de Ocosingo con la oferta turística regional. Agricultura, Sociedady Desarrollo, 13(1), 131-149. Recuperado de http://www.redalyc.org/articulo. $\underline{\text { oa? } \mathrm{id}=360545634008}$

Bessiere, J. (1998). Local Development and Heritage: Traditional Food and Cuisine as Tourist Attractions in Rural Areas. Sociologia Ruralis, 38(1), 21-34. DOI: http://doi. org/10.1111/1467-9523.00061

Cervantes, E. F., Villegas, G. A., Cesín, V. A. y Espinoza, O. A. (2008). Los quesos mexicanos genuinos: patrimonio cultural que debe rescatarse. (Primera edición). México: Mundi-Prensa.

Escobar, R., M. C., Hernández, I. M. y Gaspar, S. D. (2012). Composición fisicoquímica y microbiológica del queso bola de Ocosingo, Chiapas. Memorias XXXVI Congreso Nacional de Buiatría.

Espejel, A., Barrera, A., Herrera, B. E. y Cuevas, V. (2016). Factores estructurales en la construcción del sistema regional de innovación de vainilla (vainilla planifolia Jacks ex Andrews) en México. Agroproductividad, 9, 74-78.

Espejel, G. A. y Ramírez, G. A. G. (2017, october). Empleo e innovación en la producción de queso añejo en el municipio de Zacazonapan. Researh Gate, 205-232.

Guerrero, L., Glaret A., Verbeke W., Sulmont, C. y Hersleth M. (2016). Innovation in traditional food productos: Does it make sense? In Ch. Galanakis (Ed.), Innovation Strategies in the Food Industry (pp. 77-89). DOI: https://doi.org/10.1016/ $\underline{\text { B978-0-12-803751-5.00005-2 }}$

Hervás S. A. (2012). El mercado del queso en México. Oficina Económica y Comercial de la Embajada de España en México.

Kristbergsson, K. y Oliveira, J. (2016). Traditional foods: General and Consumer Aspects. Springer. DOI: http://doi.org/10.1007/978-1-4899-7648-2

Kühne, B., Vanhonacker, F., Gellynck, X. y Verbeke, W. (2010). Innovation in traditional food products in Europe: Do sector innovation activities match consumers' acceptance? Food Quality and Preference, 21(6), 629-638. DOI: http://doi.org/10.1016/j. foodqual.2010.03.013 
Muñoz, R. M., Altamirano, C. J. R., Aguilar, Á. J., Rendón, M. R. y Espejel, G. A. (2007). Innovación: motor de la competitividad agroalimentaria. Políticas y estrategias para que en México ocurra. UACh - CIESTAAM.

Poméon, T. (2011). De la retórica a la práctica del patrimonio: procesos de calificación de los quesos tradicionales mexicanos. (Tesis de doctorado en problemas económico-agroindustriales). México: CIESTAAM-UACH.

Rendón, M. R., Aguilar, Á. J., Muñoz, R. M. y Altamirano, G. J. R. (2007). Identificación de actores clave para la gestión de la innovación: el uso de redes sociales. Chapingo, estado de México: Universidad Autónoma Chapingo-Ciestaam/PIIAI.

Rizo, M. P., Frigant, N. y Jalba, V. (2013). Las indicaciones geográficas. Ginebra, Suiza: Organización Mundial de la Propiedad Intelectual - OMPI. Recuperado de http:// www.wipo.int/edocs/pubdocs/es/geographical/952/wipo_pub_952.pdf

Sarkar, S. y Costa, A. I. A. (2008). Dynamics of open innovation in the food industry. Trends in Food Science and Technology, 19(11), 574-580. DOI: http://doi.org/10.1016/j. tifs.2008.09.006

Stolzenbach, S., Bredie, W. L. P. y Byrne, D. V. (2013). Consumer concepts in new product development of local foods: Traditional versus novel honeys. Food Research International, 52(1), 144-152. DOI: http://doi.org/10.1016/j.foodres.2013.02.030

Trichopoulou, A., Vasilopoulou, E., Georga, K., Soukara, S. y Dilis V. (2006). Traditional foods: Why and how to sustain them. Trends in food Science and Tecnhology 17(9), 498504. DOI: https://doi.org/10.1016/j.tifs.2006.03.005

Vanhonacker, F., Kühne, B., Gellynck, X., Guerrero, L., Hersleth, M. y Verbeke, W. (2013). Innovations in traditional foods: Impact on perceived traditional character and consumer acceptance. Food Research International, 54(2), 1828-1835. DOI: http:// doi.org/10.1016/j.foodres.2013.10.027

Vendruscolo, R., Tomé da Cruz, F. y Schneider, S. (2016). (Re)valorización de los alimentos de la agricultura familiar: límites y particularidades de las estrategias agroalimentarias en el estado de Rio Grande Do Sul, Brasil. Agroalimentaria, 22(42), 149-169. Recuperado de http:/ / www.redalyc.org/articulo.oa?id=199245407009

Villegas, G. A. Z., Santos, M. A. y Cervantes, E. F. (2016). Los quesos mexicanos tradicionales. (Primera edición). Universidad Autónoma Chapingo. 